\title{
THREE SPECIES OF THE GENUS DRASSYLLUS (ARANEAE: GNAPHOSIDAE) FROM JAPAN ${ }^{1)}$
}

\author{
Takahide Kamura
}

\author{
Laboratory of Entomology, Faculty of Agriculture, Kyoto Prefectural \\ University, Shimogamo, Kyoto 606, Japan
}

\section{Synopsis}

Kamura, Takahide (Laboratory of Entomology, Faculty of Agriculture, Kyoto Prefectural University, Shimogamo, Kyoto 606, Japan): Three species of the genus Drassyllus (Araneae: Gnaphosidae) from Japan. Acta arachnol., 35: 7788 (1987).

The occurrence of the genus Drassyllus in Japan is confirmed. The male of Drassyllus sanmenensis PLATnICK et Song is described for the first time. Two new species, $D$. sasakawai and $D$. yaginumai are described.

The Zelotes complex is a large group in the family Gnaphosidae. The members of this complex are characterized by having the third and fourth metatarsi each with a preening comb on ventrodistal end (BERLAND, 1919, Fig. 1; Kaston, 1978, Fig. 527 ; Platnick \& Shadab, 1982a, Figs. 1, 2; Kamura, 1984, Fig. 11). The genus Drassyllus was originally established by CHAMBERLIN (1922) based on the American species, differing from the genus Zelotes GisTeL, 1848 , in respect to the eye arrangement and male palpal structure. Since he treated it, American species of the Zelotes complex had been generally divided into these two genera (for example, UBICK \& RoTH, 1973; KASTON, 1978). On the other hand, all European species of this complex had been placed in the genus Zelotes in broad sense (for example, Simon, 1914 ; Reimoser, 1937 ; Locket \& Millidge, 1951). Recently, Platnick \& ShadAB (1982a, 1982b, 1983) and PlatNick \& MURPhy (1984) revised the Zelotes complex and recognized the five genera: Zelotes Gistel, 1848 ; Drassyllus Chamberlin, 1922 ; Camillina Berland 1919; Trachyzelotes Lohmander, 1944, and Urozelotes Mello-Leitão, 1938. Although the opinions of European authors (GRIMM, 1985; MERRETT et al., 1985; ROBERTS, 1985) are not yet uniform as to the criteria for separating, I

1) Contribution from Lab. Entom., Kyoto Pref. Univ., No. 216. 
prefer to regard Drassyllus as generic status according to the redefinition of the genus given by Platnick \& Shadab (1982a).

In Japan, one species, which had been regarded as conspecific with the European Zelotes praeficus (L. KocH, 1866) $)^{2}$, had been known. However, YAGINUMA (1986) pointed out that this Japanese species is not true praeficus. After my recent examination, I recognized that the Japanese species is Drassyllus sanmenensis Platnick et SonG, 1986, known from China, and that two new species of Drassyllus occur in Japan. These three species are described in the present paper.

In addition, Zelotes $x$-notatus (BöSENBERG et STRAND, 1906) seems to belong to Drassyllus judging from the structure of male palp drawn by YAGINUMA (1986, Fig. 106-6). But I hesitated to make reference to this species, because a careful examination should be done in the future.

All the type specimens are preserved in the collection of the Arachnological Society of East Asia, Ohtemon-Gakuin University, Ibaraki, Osaka.

The abbreviations used in this paper are as follows: ALE, anterior lateral eye; AME, anterior median eye; MOA, median ocular area; $p$, proventral; PLE, posterior lateral eye; PME, posterior median eye; $r$, retroventral. Eye size means the length of long axis of an eye. But the measurement of posterior median eye was made at the horizontal level.

\section{Genus Drassyllus Chamberlin, 1922}

(Japanese name: Yorime-kemurigumo-zoku)

Drassyllus Chamberlin, 1922, Proc. biol. Soc. Wash., 35: 148, 166. Type species: Dras. syllus fallens ChAMBERLIN, 1922.

Epizelotes Lohmander, 1944, Ark. Zool., 35A(16): 14 (as a subgenus of Zelotes).

Carapace longer than wide, with median furrow distinct, longitudinal. Anterior eye row slightly recurved, posterior eye row slightly procurved as seen from above. MOA longer than wide, with anterior width as wide as, or slightly narrower than the posterior. PME large, often irregular in shape, very close or contiguous to each other (Figs. 1,10). Chelicera usually with four or five teeth on promargin, with two or three teeth on retromargin. Leg formula as 4-1-2-3. Trochanters of legs without a ventral notch. Metatarsi III and IV each with a preening comb on ventrodistal end. In male, abdomen

2) Recently, this species was transferred to Drassyllus by Merrett et al. (1985). 
with dorsal scutum variable from one-third to a half of abdomen in length. Male palp with terminal apophysis divisible to basal and apical projected parts, median apophysis situated retrolaterally, embolar base large, conductor membranous, and retrolateral tibial apophysis (Figs. 2, 3).

\section{Drassyllus sanmenensis Platnick et Song, 1986}

(Japanese name: Ebicha-yorime-kemurigumo)

Figs. 1-5

Zelotes praeficus: Nishikawa \& Kawanaka, 1973, Biological survey of the Mt. Hiruzen area, p. 238, fig. 28.-Yaginuma, 1973, Fac. Let. Rev. Otemon Gakuin Univ., (7) : $71 ; 1977$, Acta arachnol., 27 (special no.) : 404.

Zelotes sp.: YAginuma, 1986, Spiders of Japan in Color, p. 192 (1987, Corrigenda, p. 4). Drassyllus sanmenensis Platnick \& Song, 1986, Amer. Mus. Novitates, (2848) : 17, figs.

67, 68 (female holotype from China, in Institute of Zoology, Academia Sinica, Beijing, China, examined).

Measurements of $1 \hat{\delta}$ and 1 ㅇ (in $\mathrm{mm}$; $\} /$ 우). Body length 6.30/7.10. Carapace length 2.90/2.95, width 2.25/2.30. Abdomen length $3.40 / 4.15$, width $1.75 / 2.40$.

Length of legs :

$\begin{array}{ccccccc}\text { Leg } & \text { Femur } & \text { Patella } & \text { Tibia } & \text { Metatarsus } & \text { Tarsus } & \text { Total } \\ \text { I } & 2.20 / 2.38 & 1.33 / 1.47 & 1.73 / 1.83 & 1.53 / 1.67 & 1.05 / 1.32 & 7.84 / 8.67 \\ \text { II } & 1.90 / 2.00 & 1.10 / 1.17 & 1.40 / 1.47 & 1.40 / 1.43 & 1.10 / 1.13 & 6.90 / 7.20 \\ \text { III } & 1.70 / 1.77 & 0.88 / 0.90 & 1.12 / 1.17 & 1.37 / 1.45 & 0.87 / 0.97 & 5.94 / 6.26 \\ \text { IV } & 2.40 / 2.55 & 1.30 / 1.33 & 1.87 / 2.00 & 2.30 / 2.40 & 1.10 / 1.20 & 8.97 / 9.48\end{array}$

Eye sizes and interdistances: AME 0.13/0.13, ALE 0.15/0.15, PME 0.13/0.14, PLE 0.14/0.14; AME-AME 0.05/0.05, AME-ALE 0.01/0.01, PME-PME 0.03/0.03, PME-PLE 0.05/0.07, ALE-PLE 0.08/0.08. MOA anterior width $0.28 / 0.29$, posterior width $0.28 / 0.30$, length $0.35 / 0.36$. Clypeus height $0.13 / 0.13$.

Variation (in mm). Body length $\$ 5.40-6.80$, ㅇ 4 4.55-8.20. Carapace length ô 2.50-3.20, 우 2.05-3.35; width ô 1.90-2.50, 우 1.60-2.50.

Ventral spines on legs I and II. 오, 令. Tibia: I $0-0-0$ (rarely $0-1 \mathrm{r}-0$ ), II $0-1 \mathrm{r}-0$ or $1 \mathrm{r}-1 \mathrm{r}-0$; metatarsus: I and II $2-0-0$ or $2-1 \mathrm{p}-0$ (rarely $2-2-0$ ).

Eye arrangement (Fig. 1): PME slightly separated from each other. Male palp (Figs. 2, 3): projection of terminal apophysis with a distal notch; median apophysis with a retrolateral projection on posterior part. Epigynum (Fig. 4): 

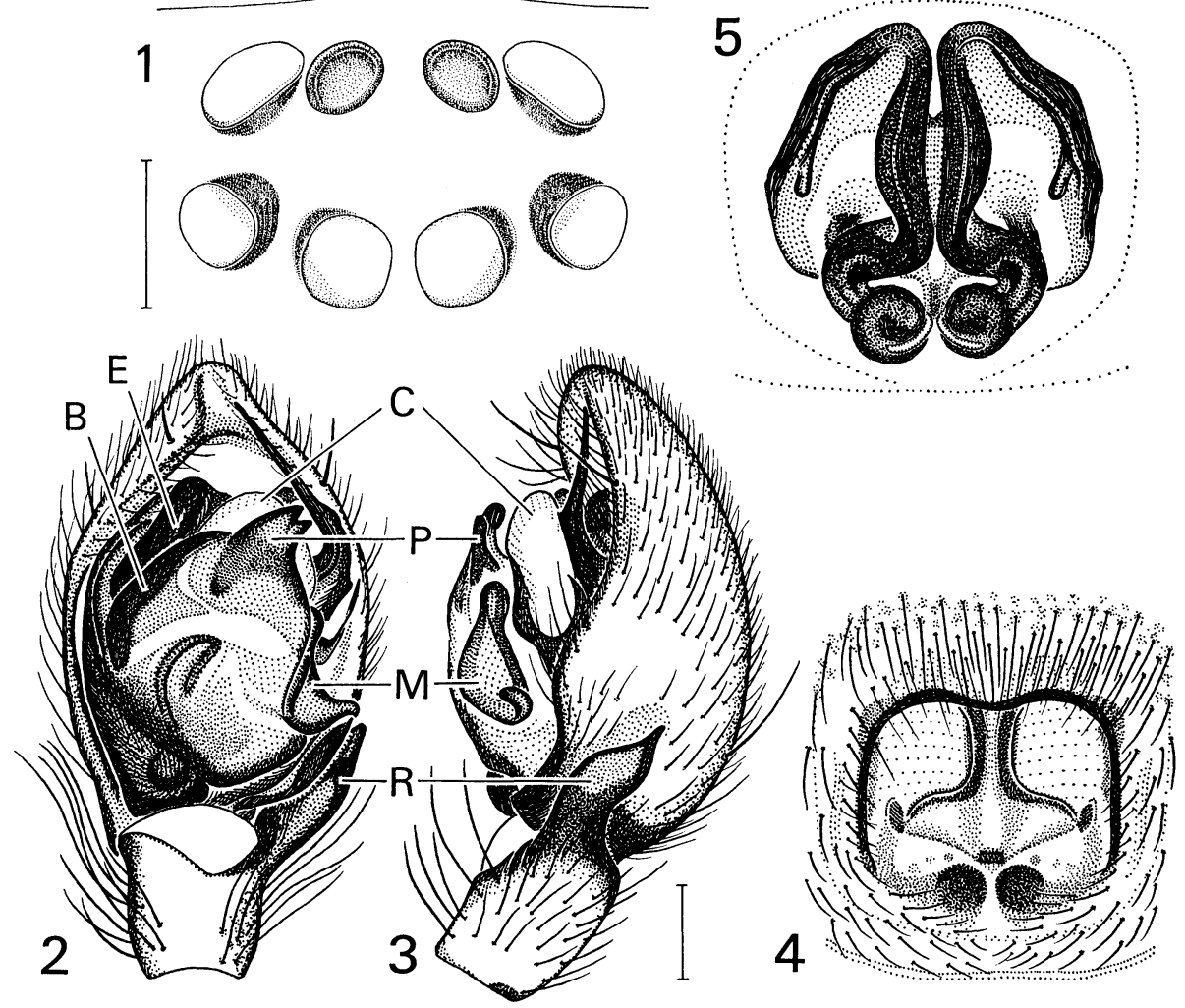

Figs. 1-5. Drassyllus sanmenensis PlAtnick et Song, 1986. 1. Eye area, dorsal view. 2. Left male palp, ventral view. 3. Ditto, retrolateral view. 4. Epigynum, ventral view. 5. Female genitalia, dorsal view. Abbreviations : B, base of terminal apophysis; $\mathrm{C}$, conductor; E, embolar base; $\mathrm{M}$, median apophysis; $\mathrm{P}$, projection of terminal apophysis; $R$, retrolateral tibial apophysis. (Scale : $0.2 \mathrm{~mm}$.)

roughly square, slightly wider than long. Female genitalia as shown in Fig. 5.

Colour. Cephalothorax and appendages generally dark reddish brown; endites and coxae of legs paler than labium and sternum; lateral surfaces of femora and dorsal surfaces of patellae of legs paler; metatarsi and tarsi of legs yellowish to pale reddish brown. Abdomen dark brown. Spinnerets dark reddish brown. Male abdomen with dorsal scutum dark reddish brown.

Specimens examined. 우 (holotype), Sanmen, Zhejiang, China, 20. IV. 1978 (S. X. Zheng). Japan: Hokkaido: 1우, Mt. Rausu-dake, 5-15. VIII. 1963 (O. SATo). Yamagata Pref. : 1\}, Miyauchi-cho, 4. VI. 1961 (S. NISHIKI); 1\}, Higa- 
shine-shi, 3. VI. 1986 (H. YoshidA). Niigata Pref. : 1 옹 Sadogashima Is., 4. VI. 1958 (N. ITo). Mie Pref. : 2\}, Tôshi-jima Is., Toba-shi, 2. VI. 1973 (O. TomINAGA); 1ో, Yutanigawa, Iitaka-cho, linan-gun, $250 \mathrm{~m}$ alt., 5-6. VI. 1982 (O. ToMINAGA). Shiga Pref. : 2 우, Gokashô-cho, Kanzaki-gun, 24. VII. 1979 (T. YAMANO); 1 우 1\}, Shigaraki-cho, Kôga-gun, 25. V-1. VI. 1985 (A. HoGA). Kyoto Pref.: 1\}, Hitsukura-dani, Ashû, Miyama-cho, Kitakuwata-gun, 15. V. 1983 (T. KAMURA); 1\}, Sugô, Ashû, $380 \mathrm{~m}$ alt., L. C. [C3543-3518] $]^{3)}$, 27. V. 1985 (T. KAMURA); 41 우, 145\}, east of Midorogaike Pond, Matsugasaki, Kyoto-shi, $80 \mathrm{~m}$ alt., L. C. [C3546-3503], IV-VII. 1982-1984 (T. Kamura); 1\}, Mt. Arashiyama, Kyoto-shi, L. C. [C3540-3500], 16.X. 1986 (T. KAMURA), collected as subadult, matured on 25. XI. 1986 ; 2 우, Mt. Otowa-yama, Kyoto-shi, L. C. [C3551-3458], 21. VI-12. VII. 1986 (A. HoGA); 1\}, vicinity of Kutsuwa-ike Pond, Ujitawara-cho, Tsuzuki-gun, 200-260 m alt., L. C. [C3550-3451], 25. V-1. VI. 1985 (A. HoGA); 1 오, Kasagi-cho, Sôraku-gun, $420 \mathrm{~m}$ alt., L.C. [C3557-3447], 22-29. VII. 1985 (S. KANENO); 1 우, Minamiyamashiro-mura, Sôraku-gun, 280-320 m alt., L. C. [C3559-3444], 23. VII. 1985 (T. Kamura) ; 4 우, Kamo-cho, Sôraku-gun, 260-280 m alt., L. C. [C35543444], 9. VI. 1985 (T. Kamura). Osaka Pref. : 1 우 , Hozumidai, Ibaraki-shi, $30 \mathrm{~m}$ alt., L.C. [C3533-3448], 28. VI. 1983 (Y. Nishikawa). Hyôgo Pref. : 1\}, Kurakuen, Nishinomiya-shi, L. C. [C3518-3445], 23. V. 1986 (H. SHImizU). Nara Pref. : 2\}, from Ichinoi to Byakugôji-cho, Nara-shi, 29. V-6. VI. 1976 (O. TominaGA); 1ิ̂, Nara-shi, 15. X.1986 (S. KANENO), collected as subadult, matured on 27. XI. 1986 ; 2\}, Ôgi, Tsuge-mura, Yamabe-gun, 29. V.1979 (T. Yamano). Tottori Pref.: 1우, Inoko, Tottori-shi, 15. IX.1963 (T. ARITA). Yamaguchi Pref.: 1우, Torigoe, 30. VII. 1961 (K. OJIMA). Ehime Pref.: 5今, Ikata-cho, Nishiuwa-gun, 10. V. 1982 (T. YAmano). Kôchi Pref.: 2우, Shirahama, Ôtsuki-cho, Hata-gun, 18. VII. 1982 (T. YAMANo). Fukuoka Pref. : 1ㅇ, Mt. Hikosan, 4-9. VIII. 1958 (C. OKuma); 11우, 2\}, ibid., 27-31. V. 1959 (C. OKuma); 4오, 23\}, Mt. Tachibanayama, Fukuoka-shi, 6. V-3. VI. 1979 (K. YAmAGISHI).

Distribution. Japan (Hokkaido, Honshu, Shikoku, Kyushu), China.

Remarks. D. sanmenensis had been known as the European Zelotes praeficus (L. Koch, 1866) in Japan (NishikAwA \& KawanaKA, 1973; Yaginuma, 1973, 1977) until YAGINUMA (1986) pointed out its misidentification. The both species are somewhat similar to each other in the shape of epigynum, but $D$. sanmenensis is distinguished from $Z$. praeficus by having a distal notch on the projection of

3) Matsumoto's locality code (Matsumoto, 1979). 
terminal apophysis in male palp.

\section{Drassyllus sasakawai n. sp.}

(Japanese name: Yama-yorime-kemurigumo)

Figs. 6-9

Measurements of holotype and allotype (in mm; 今/우). Body length 5.85/ 7.85. Carapace length $2.60 / 3.00$, width $2.10 / 2.40$. Abdomen length $3.25 / 4.85$, width $1.90 / 2.85$.

Length of legs :

$\begin{array}{ccccccc}\text { Leg } & \text { Femur } & \text { Patella } & \text { Tibia } & \text { Metatarsus } & \text { Tarsus } & \text { Total } \\ \text { I } & 2.10 / 2.40 & 1.23 / 1.43 & 1.67 / 1.92 & 1.53 / 1.72 & 1.22 / 1.35 & 7.75 / 8.82 \\ \text { II } & 1.78 / 2.08 & 1.03 / 1.23 & 1.32 / 1.53 & 1.28 / 1.50 & 1.07 / 1.18 & 6.48 / 7.52 \\ \text { III } & 1.55 / 1.82 & 0.83 / 1.00 & 1.05 / 1.25 & 1.25 / 1.52 & 0.82 / 0.98 & 5.50 / 6.57 \\ \text { IV } & 2.20 / 2.57 & 1.13 / 1.40 & 1.72 / 2.07 & 2.03 / 2.53 & 0.98 / 1.23 & 8.06 / 9.80\end{array}$

Eye sizes and interdistances: AME 0.10/0.13, ALE 0.13/0.14, PME 0.13/0.13, PLE 0.11/0.12; AME-AME 0.05/0.05, AME-ALE 0.01/0.01, PME-PME 0.03/0.04, PME-PLE 0.04/0.05, ALE-PLE 0.07/0.08. MOA anterior width $0.24 / 0.28$, posterior width $0.28 / 0.29$, length $0.33 / 0.35$. Clypeus height $0.13 / 0.11$.

Variation (in mm). Body length $\$ 5.20-7.05$, 우 5.60-7.90. Carapace length o 2.45-3.30, 우 2.50-3.05; width ô 1.95-2.65, 우 1.85-2.40.

Ventral spines on legs I and II. 우, $\hat{\circ}$. Tibia: I $0-1 \mathrm{r}-0$ or $1 \mathrm{r}-1 \mathrm{r}-0$ (rarely $0-0-0$ or $1 \mathbf{r}-2-0)$, II $1 \mathbf{r}-1 \mathbf{r}-0$ or $1 \mathbf{r}-2-0$ (rarely $0-1 \mathbf{r}-0)$; metatarsus : I and II $2-2-0$ (rarely $2-1 \mathrm{p}-0$ or $2-1 \mathrm{r}-0$ ).

Eye arrangement similar to that of $D$. sanmenensis Platnick et Song. Male palp (Figs. 6,7): projection of terminal apophysis with a small pointed projection on retrolateral side; median apophysis very large, without a retrolateral projection. Epigynum (Fig. 8): roughly square, longer than wide. Female genitalia as shown in Fig. 9.

Colour. Very similar to D. sanmenensis Platnick et Song.

Type-series. Holotype: $\hat{\delta}$, Sugô, Ashû, Miyama-cho, Kitakuwata-gun, Kyoto Pref., Japan, $380 \mathrm{~m}$ alt., L.C. [C3543-3518], 26-28. V. 1984, pitfall trap (T. KAMURA). Allotype: 우, southern slope of Mt. Taiko-yama, Yasaka-cho, Yosa-gun, Kyoto Pref., $620 \mathrm{~m}$ alt., L. C. [C3512-3541], 8. VII. 1982 (T. KamURA). Paratypes: $2 \hat{\delta}$, same data as holotype; $1 \hat{\delta}$, Obana-dani, Hirogawara, Kyoto-shi, 

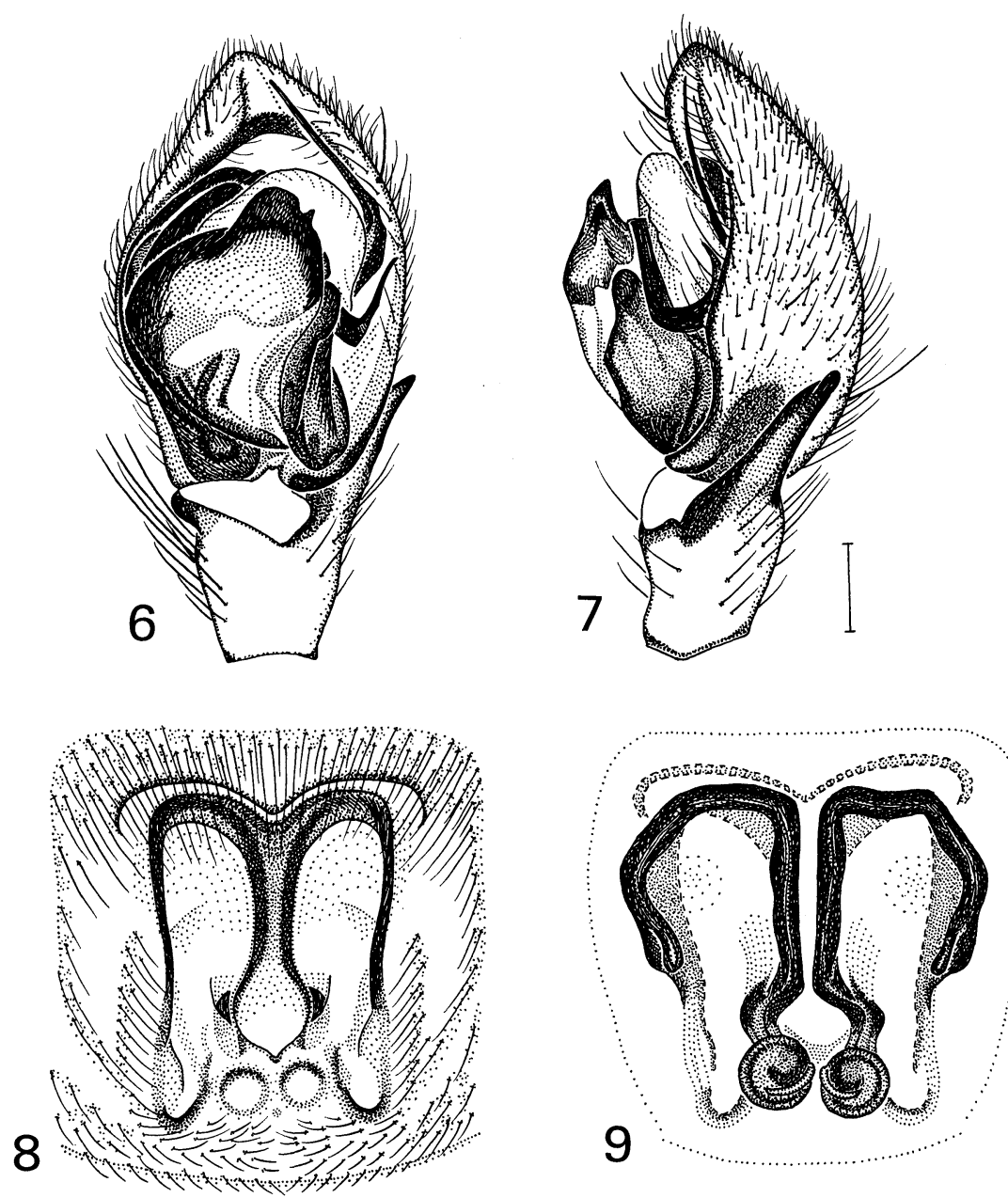

Figs. 6-9. Drassyllus sasakawai n. sp. 6. Left male palp, ventral view. 7. Ditto, retrolateral view. 8. Epigynum, ventral view. 9. Female genitalia, dorsal view. (Scale : $0.2 \mathrm{~mm}$. )

Kyoto Pref., $520 \mathrm{~m}$ alt., L. C. [C3544-3515], 23. V. 1981 (T. KamURA); 1\}, eastern slope of Mt. Tsuzumigadake, Miyazu-shi, Kyoto Pref., $520 \mathrm{~m}$ alt., L. C. [C35113536], 27. V. 1982 (T. Kamura); 1 ㅇ, southern slope of Mt. Koshio-yama, Kyotoshi, Kyoto Pref., 500-560 m alt., L.C. [C3538-3457], 3. IV. 1986 (S. KANENo), collected as subadult, matured on 23. IV.1986; 1우 18\}, ibid., 1.V.1986 (S. KANENO \& T. KAMURA), 1 female was collected as subadult, matured on 6 . V. 
1986.

Other specimens examined. Aomori Pref. : 1우, Yunokawa-onsen, Kawauchimachi, Shimokita-gun, $200 \mathrm{~m}$ alt., 28-29. VIII. 1975 (O. TominAGA). Yamagata Pref. : 1우, Higashine-shi, 25. V.1986 (H. YoshidA). Gifu Pref. : 3\}, Kiriyama, Nyûkawa-mura, Ôno-gun, 4. V.1973 (K. Katsura \& O. TominaGa); 1\}, Sugatani, Horado-mura, Mugi-gun, $180 \mathrm{~m}$ alt., L. C. [C3648-3535], 30. V. 1985 (T. ZAKo). Mie Pref.: 1§, Nagano-tôge, Ôyamada-mura, Ayama-gun, $450 \mathrm{~m}$ alt., 1. VI. 1973 (K. Katsura \& O. Tominaga). Shiga Pref.: 1\}, Shigaraki-cho, Kôga-gun, 25. V-1. VI. 1985 (A. HogA); 3今, Miyajiri, Shigaraki-cho, 240-300 m alt., L. C. [C3558-3452], 17-31. V. 1986 (A. HoGA); 1ㅇ, 2\}, ibid., 31. V-6. VI. 1986 (A. HogA). Kyoto Pref.: 1\}, Ashû, Miyama-cho, Kitakuwata-gun, 15. V. 1982 (T. Kamura); 1 오 Sugô, Ashû, $400 \mathrm{~m}$ alt., L. C. [C3542-3518], 14-16. V. 1983 (T. KAmURA); 11\}, southern slope of Mt. Koshio-yama, Kyoto-shi, 500$560 \mathrm{~m}$ alt., L.C. [C3538-3457], 3.IV.1986 (S. KANENO), collected as subadults, matured on 11-16. IV.1986; 1우, Mt. Otowa-yama, Kyoto-shi, L. C. [C3551-3458], 14-28. VI. 1986 (A. HogA); 11우, ibid., 5. VII-9. VIII. 1986 (A. HoGA). Osaka

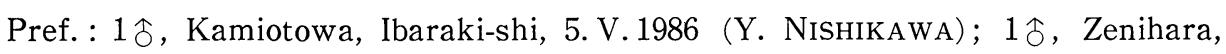
Ibaraki-shi, $440 \mathrm{~m}$ alt., 20. V.1978 (Y. NishikAWA). Hyôgo Pref.: 2\}, Ikuno, Dôjô-cho, Kôbe-shi, 150-230 m alt., 6. VI. 1981 (Y. NisHikawa). Nara Pref. : $3 \hat{\delta}$, Ôgi, Tsuge-mura, Yamabe-gun, 29. V.1979 (T. YAMANO). Fukuoka Pref. : 1 우, Mt. Hikosan, 27-31. V. 1959 (C. OKuma). Kumamoto Pref.: 1우, Kikuchi-shi, 27. IV. 1979 (H. MAKIHARA).

Distribution. Japan (Honshu, Kyushu).

Remarks. This new species is somewhat similar to D. yaginumai $\mathrm{n}$. $\mathrm{sp}$. in the shape of terminal apophysis in male palp, but is easily distinguished by its large median apophysis without a retrolateral projection.

Etymology. Specific name is dedicated to Dr. Mitsuhiro SaSAKawa.

\section{Drassyllus yaginumai n. sp.}

(Japanese name: Hime-yorime-kemurigumo)

Figs. $10-14$

Measurements of holotype and allotype (in mm; $1 /$ o ). Body length 5.10/ 5.35. Carapace length $2.30 / 2.45$, width $1.85 / 1.90$. Abdomen length 2.80/2.90, width $1.50 / 1.60$. 
Length of legs :

$\begin{array}{ccccccc}\text { Leg } & \text { Femur } & \text { Patella } & \text { Tibia } & \text { Metatarsus } & \text { Tarsus } & \text { Total } \\ \text { I } & 1.83 / 1.94 & 1.09 / 1.13 & 1.38 / 1.41 & 1.26 / 1.33 & 1.05 / 1.10 & 6.61 / 6.91 \\ \text { II } & 1.53 / 1.65 & 0.89 / 0.96 & 1.08 / 1.15 & 1.08 / 1.16 & 0.90 / 0.98 & 5.48 / 5.90 \\ \text { III } & 1.38 / 1.46 & 0.73 / 0.80 & 0.91 / 0.98 & 1.08 / 1.19 & 0.73 / 0.84 & 4.83 / 5.27 \\ \text { IV } & 1.99 / 2.11 & 1.05 / 1.13 & 1.55 / 1.65 & 1.79 / 1.93 & 0.93 / 1.05 & 7.31 / 7.87\end{array}$

Eye sizes and interdistances: AME 0.13/0.13, ALE 0.16/0.18, PME 0.16/0.18, PLE 0.14/0.13; AME-AME 0.05/0.05, AME-ALE 0.01/0.01, PME-PME 0.00/0.00, PME-PLE 0.04/0.05, ALE-PLE 0.06/0.08. MOA anterior width $0.28 / 0.29$, posterior width $0.32 / 0.36$, length $0.39 / 0.39$. Clypeus height $0.11 / 0.13$.

Variation (in mm). Body length $\hat{0} 4.75-5.85$, 우 5.25-7.15. Carapace length 今 2.20-2.55, 우 2.25-3.10; width ㅇํ $1.70-2.05$, 우 1.65-2.45.

Ventral spines on legs I and II. 우, $\delta$. Tibia: I $0-0-0$, II $0-0-0$ or $0-1 \mathrm{r}-0$ (rarely $1 \mathrm{r}-1 \mathrm{r}-0)$; metatarsus: I and II 2-0-0.

Eye arrangement (Fig. 10): PME entirely contiguous to each other. Male palp (Figs. 11, 12): projection of terminal apophysis with a small pointed projection on retrolateral side; median apophysis with a retrolateral projection on posterior part. Epigynum (Fig. 13): narrowed anteriorly, longer than wide. Female genitalia as shown in Fig. 14.

Colour. Similar to D. sanmenensis Platnick et SoNG, but more paler.

Type-series. Holotype: $\hat{\jmath}$, allotype: $ᄋ$, moor in Midorogaike Pond, Kamigamo, Kyoto-shi, Kyoto Pref., Japan, $75 \mathrm{~m}$ alt., L. C. [C3546-3503], 30. V-10. VI. 1985, pitfall trap (T. KamURA). Paratypes: 2个, 16-28. VI. 1982; 2ㅇ, 28. VI-5. VII. 1982; 1ㅇ, 23. VIII-6. IX. 1982;1\}, 4-15. VI. 1983;1\}, 1-11. VI. $1984 ; 1$ 우, 1-13. VIII. $1985 ; 1$ 우, 13-22. VIII. 1985 . The locality and collector of all paratypes are same as holotype.

Other specimens examined. 1ㅇ, same locality as holotype, 21-22. VII. 1979 (A. Noto); 4 우 ibid., 21. VII. 1979 (Y. NishikAWA); 1 우, ibid., 18. VII. 1980 (H. TANAKA); 1 우, ibid., 9-16. VI. 1982 (T. KAMURA); 1우, ibid., 23-30. VII. 1982 (T. KAMURA); 1우, 1§, ibid., 28. VI-10. VII. 1984 (T. KAMURA); 1우, ibid., 8-15. VII. 1985 (T. Kamura). 1우, farm of Kyoto Pref. Univ., Shimogamo, Kyoto-shi, Kyoto Pref., $70 \mathrm{~m}$ alt., L. C. [C3546-3502], 5. VI. 1984 (A. UEDA) ; 1 우, ibid., 24. VII. 1985 (A. UEDA).

Distribution. Japan (Honshu: Kyoto Pref.).

Remarks. This new species is closely related to D. coreanus PAIK, 1986, 

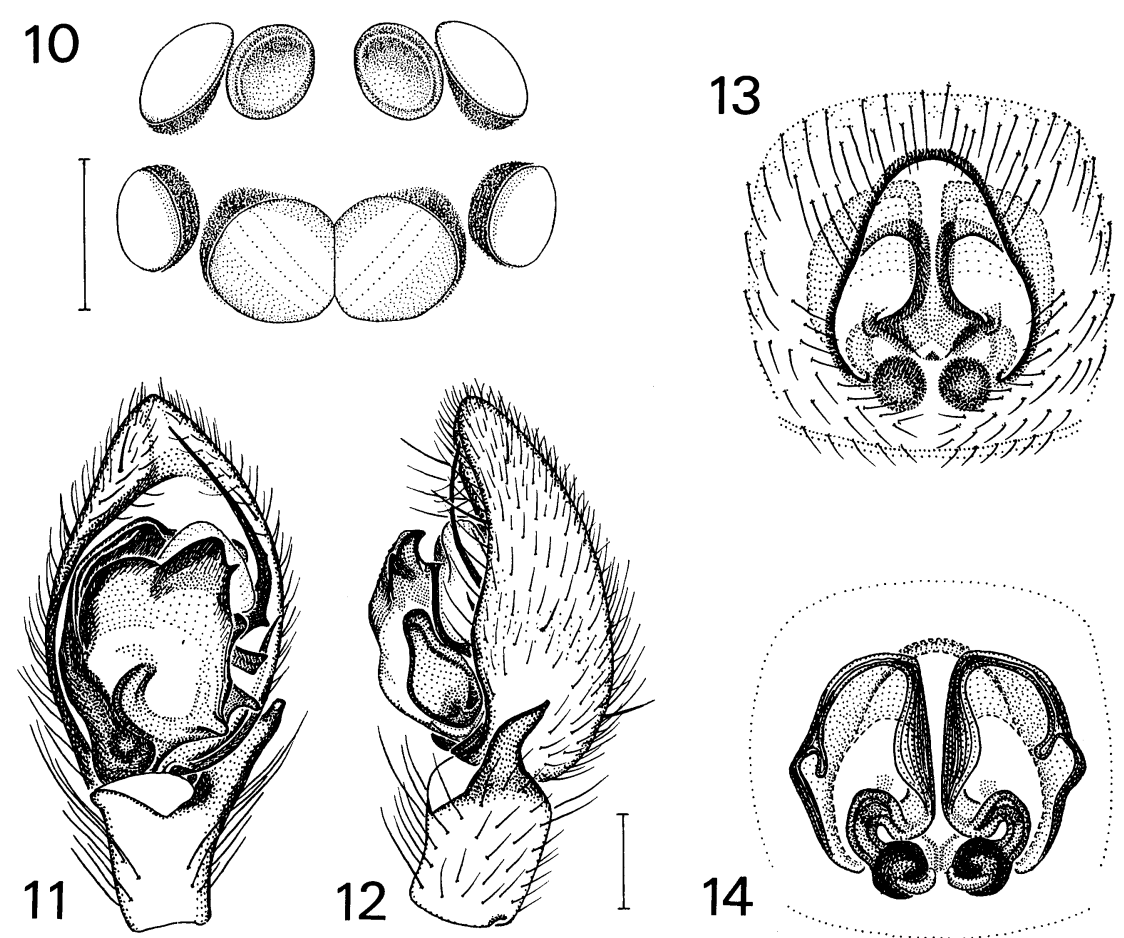

14

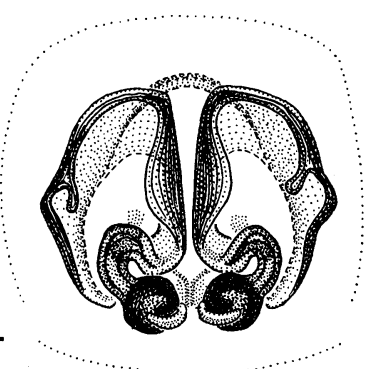

Figs. 10-14. Drassyllus yaginumai n. sp. 10. Eye area, dorsal view. 11. Left male palp, ventral view. 12. Ditto, retrolateral view. 13. Epigynum, ventral view. 14. Female genitalia, dorsal view. (Scale : $0.2 \mathrm{~mm}$.)

known from Korea, in the structure of male palp, but is separated by having the entirely contiguous PME, and not having stiff bristles on dorsal surface of male palpal tibia.

Etymology. Specific name is dedicated to Dr. Takeo Yaginuma.

\section{Acknowledgements}

I wish to express my hearty thanks to Dr. M. SASAKawa, Kyoto Prefectural University, and Dr. T. Yaginuma, Ohtemon-Gakuin University, Osaka, for their constant guidance and critical reading the manuscript, and to Dr. K. Y. PAIK, Kyungpook University, Taegu, Korea, and Dr. D.X. Song, Institute of Zoology, Academia Sinica, Beijing, for their loaning valuable specimens. My 
thanks are also due to Dr. Yaginuma, Miss C. Okuma and Messrs. A. Hoga, S. Kaneno, Y. Nishikawa, H. Shimizu, A. Ueda, T. Yamano, H. Yoshida and T. ZAKO for their offering or loaning specimens used in this study, and to Mr. T. HAYASHI for sending a copy of a literature. I am also grateful to Section for Protection of Cultural Properties, Department of Cultural Affairs and Tourism, Kyoto City Government for permitting me to collect material in the moor of Midorogaike Pond.

摘 要

加村隆英（京都府立大学農学部昆虫学研究室, ү606 京都市左京区下鴨半木町）：日本産ヨリメ ケムリグモ属（新称）Drassyllus（真正クモ目：ワシグモ科）の 3 種。

日本に护けるDrassyllus 属の分布を確認した。エビチャヨリメケムリグモ（改称）D. sanmenensis PLATNICK et SONG の雄をはじめて記載し, 2 新種, ヤマヨリメケムリグモ（新称） D. sasakawai とヒメヨリメケムリグモ（新称）D. yaginumaiを記載した。Drassyllus 属は広 義の Zelotes 属に含められることがあるが，筆者は Drassyllus 属を独立属として扱った。

\section{References}

Berland, M.L., 1919. Note sur le peigne métatarsal que possèdent certaines araignées de la famille des Drassidae. Bull. Mus. Hist. nat., Paris, (6) : 458-463.

Bösenberg, W. \& E. Strand, 1906. Japanische Spinnen. Abh. senck. naturf. Ges., 30 : 93-422, pls. 3-16.

Chamberlin, R. V., 1922. The North American spiders of the family Gnaphosidae. Proc. biol. Soc. Wash., 35 : 145-172.

Gistel, J., 1848. Naturgeschichte des Thierreichs für höhere Schulen. pp. i-xvi+1-220, 32 pls. Stuttgart.

Grimm, U., 1985. Die Gnaphosidae Mitteleuropas (Arachnida, Araneae). Abh. Naturwiss. Ver. Hamburg, (NF), $26: 1-318$.

Kamura, T., 1984. Notes on Japanese gnaphosid spiders (I). Atypus, (85) : 1-8. (In Japanese.)

Kaston, B. J., 1978. How to Know the Spiders (third ed.). pp. i-viii+1-272. Wm. C. Brown Co., Dubuque.

KосH, L., 1866. Die Arachnidenfamilie der Drassiden, 1. pp. 1-191. Nürnberg.

Locket, G.H. \& A.F. Millidge, 1951. British Spiders, 1. pp. i-x+1-310. Ray Society, London.

Lohmander, H., 1944. Vorläufige Spinnennotizen. Ark. Zool., 35A (16) : 1-21.

Matsumoto, S., 1979. Locality code as a method indicating the geographical point in the eight-figured number. Bull. biogeogr. Soc. Japan, 34:21-27. (In Japanese, with English summary.)

Mello-Leitão, C.F. de, 1938. Algunas arañas nuevas de la Argentina. Rev. Mus. La 
Plata, New ser., Zool., 1: 89-118.

Merrett, P., G. H. Locket \& A.F. Millidge, 1985. A check list of British spiders. Bull. Br. arachnol. Soc., $6: 381-403$.

Nishikawa, Y. \& Y. Kawanaka, 1973. Spiders of the Hiruzen Area, Okayama Pref.

Biological Survey of the Mt. Hiruzen Area (Okayama Pref.), Otemon-Gakuin University Biological Club. pp. 207-243. (In Japanese.)

PAIK, K. Y., 1986. Korean spiders of the genus Drassyllus (Araneae; Gnaphosidae). Korean Arachnol., $2: 3-13$.

Platnick, N.I. \& J.A. Murphy, 1984. A revision of the spider genera Trachyzelotes and Urozelotes (Araneae, Gnaphosidae). Amer. Mus. Novitates, (2792) : 1-30.

Platnick, N.I. \& M.U. Shadab, 1982a. A revision of the American spiders of the genus Drassyllus (Araneae, Gnaphosidae). Bull. Amer. Mus. nat. Hist., 173: 1-97.

- \& - 1982b. A revision of the American spiders of the genus Camillina (Araneae, Gnaphosidae). Amer. Mus. Novitates, (2748) : 1-38.

$\&-1983$. A revision of the American spiders of the genus Zelotes (Araneae, Gnaphosidae). Bull. Amer. Mus. nat. Hist., 174: 97-192.

Platnick, N. I. \& D.X. Song, 1986. A review of the zelotine spiders (Araneae, Gnaphosidae) of China. Amer. Mus. Novitates, (2848) : 1-22.

Reimoser, E., 1937. Familie: Gnaphosidae oder Plattbauchspinnen. Die Tierwelt Deutschlands, 33. pp. 1-41. Jena.

Roberts, M. J., 1985. The Spiders of Great Britain and Ireland, 1. pp. 1-229. Harley Books, Essex.

Simon, E., 1914. Les Arachnides de France, 6(1). pp. 1-308. Paris.

Ubick, D. \& V.D. Roth, 1973. Nearctic Gnaphosidae including species from adjacent Mexican states. Amer. Arachnol., (9), suppl. 2: 1-12; suppl. 3:1-6.

Yaginuma, T., 1973. The spider fauna of Japan (III). Fac. Let. Rev. Otemon Gakuin Univ., (7) : 69-74. (In Japanese.)

- 1977. A list of Japanese spiders (revised in 1977). Acta arachnol., 27 (special no.) : 367-406. (In Japanese, with English synopsis.)

, 1986. Spiders of Japan in Color, new edition. pp. i-xxiv+1-305, pls. 1-64. Hoikusha, Osaka. (In Japanese.)

, 1987. Corrigenda of "Spiders of Japan in Color, 1986", pp. 1-4. (In Japanese.) 